\title{
No effect of fenugreek, bergamot and olive leaf extract on glucose homeostasis in patients with prediabetes: a randomized double-blind placebo-controlled study
}

\author{
Matilda Florentin, Evangelos Liberopoulos, Moses S. Elisaf, Vasilis Tsimihodimos
}

Department of Internal Medicine, Medical School of Ioannina, University of Ioannina, Ioannina, Greece

Submitted: 28 June 2019

Accepted: 30 June 2019

Arch Med Sci Atheroscler Dis 2019; 4: e162-e166

DOI: https://doi.org/10.5114/amsad.2019.86756

Copyright @ 2019 Termedia @ Banach

\section{Abstract}

Introduction: The main pathophysiologic mechanism of type 2 diabetes (T2D) is insulin resistance, which exists several years before T2D diagnosis. The term 'prediabetes' applies to patients with insulin resistance but without overt T2D. The improvement of glucose homeostasis in these patients may prevent or delay the development of T2D and its complications. Data suggest that fenugreek, olive leaf polyphenols and bergamot extract may improve carbohydrate metabolism. We examined the effect of an agent containing fenugreek, olive leaf polyphenols and bergamot extract (active agent) on glucose homeostasis in patients with prediabetes.

Material and methods: This was a single-center, randomized, double-blind, placebo-controlled trial; patients with prediabetes $(N=100)$ were randomized to treatment with the active agent or placebo. The primary efficacy endpoint was the change in glycated hemoglobin $\left(\mathrm{HbA}_{1 c}\right)$ at 6 months after treatment initiation. Secondary endpoints included changes in other parameters of glucose metabolism and lipid profile.

Results: Overall 87 patients completed the study. No significant change in $\mathrm{HbA}_{1 \mathrm{c}}$ was observed in either treatment group. Similarly, fasting plasma glucose, insulin, homeostasis model assessment-insulin resistance (HOMA-IR) index and lipid profile remained unaltered in both groups.

Conclusions: The administration of an agent containing fenugreek, olive leaf polyphenols and bergamot extract for 6 months did not improve glycemia or lipid parameters in patients with prediabetes.

Key words: glucose, insulin, lipid profile, prediabetes.

\section{Introduction}

Diabetes is a worldwide health problem reaching epidemic proportions nowadays [1]. Type 2 diabetes (T2D) accounts for $90-95 \%$ of all diagnosed cases of diabetes [1]. Complications associated with T2D may lead to functional disability, vascular complications and premature death [2]. Therefore, prevention or delay of T2D development is of major clinical importance [3].

Overt T2D is usually preceded by prediabetes, which is characterized by impaired fasting glucose (IFG), expressed as fasting plasma glucose levels between 100 and $125 \mathrm{mg} / \mathrm{dl}$, or impaired glucose tolerance (IGT), expressed as plasma glucose levels between 140 and $200 \mathrm{mg} / \mathrm{dl} 2 \mathrm{~h}$ af-

\author{
Corresponding author: \\ Matilda Florentin \\ Department \\ of Internal Medicine \\ Medical School \\ of loannina \\ University of Ioannina \\ 45110 loannina, Greece \\ Phone: +306944662406 \\ E-mail: matildaflorentin@ \\ yahoo.com
}


ter oral intake of $75 \mathrm{~g}$ of glucose [3]. IFG and IGT reflect the presence of insulin resistance, which, in turn, leads to T2D [2, 4]. Lowering plasma glucose in patients with prediabetes can effectively prevent or delay the development of T2D and its associated complications [3].

Several herbal substances seem to improve glucose metabolism. Specifically, fenugreek (Trigonella foenum-graecum L.), which includes soluble fibers, saponins, trigonelle, diosgenin and 4-hydroxy-isoleucine, has been associated with reduction both in fasting and postprandial glucose levels, as well as increase in C-peptide values [5]. Some studies and a meta-analysis demonstrated a reduction in glycated hemoglobin $\left(\mathrm{HbA}_{1 \mathrm{c}}\right)$ levels with fenugreek mainly in patients with $T 2 D$, while the doses of antidiabetic drugs were reduced in patients who took fenugreek on top of their hypoglycemic treatment $[6,7]$. Furthermore, this agent has been found to improve lipid profile, possibly by inhibiting cholesterol and bile acid absorption from the small intestine [7].

The use of olive leaf polyphenols (Olea europaea L.) has been associated with improvement in insulin sensitivity by $15 \%$ and pancreatic $\beta$-cell responsiveness by $28 \%$ in middle-aged obese men [8].

Bergamot extract may improve both hyperglycemia and lipid profile, as it was shown to reduce total and low-density lipoprotein cholesterol (LDL-C), triglycerides and small-dense LDL particles and increase high-density lipoprotein cholesterol (HDL-C) in one study [9]. It also reduced carotid intima media thickness (cIMT) in a study including patients with IGT and T2D [10].

In this context, we investigated the effects of an agent containing bergamot extract $(500 \mathrm{mg}$ ), fenugreek seed extract (200 mg) and olive leaf extract (100 mg) (active agent - AA) on glucose homeostasis in patients with prediabetes.

\section{Material and methods}

\section{Study population}

Consecutive patients with prediabetes $(N=100)$ attending the Outpatient Lipid and Obesity Clinic of the University Hospital of Ioannina, Ioannina, Greece participated in the present study. The presence of prediabetes, based on the American Diabetes Association (ADA) definition, was identified with any of the following:

- IFG (fasting plasma glucose $=100-125 \mathrm{mg} / \mathrm{dl}$ );

- IGT (plasma glucose levels between 140 and $200 \mathrm{mg} / \mathrm{dl} 2 \mathrm{~h}$ after ingesting $75 \mathrm{~g}$ of glucose); - $\mathrm{HbA}_{1 \mathrm{c}}=5.7-6.4 \%$.

Patients with overt T2D were excluded from the study. Patients taking drugs which affect glucose homeostasis (e.g. diuretics, statins, $\beta$-blockers, antipsychotics) were included in the study only if the doses of these agents were stable for the last 8 weeks, while no change in their treatment was made during the study period.

The study protocol was approved by the Ethics Committee of the University Hospital of Ioannina and all participants gave their written informed consent before enrollment.

\section{Study design}

This was a randomized, double-blind, placebo-controlled study. Subjects were randomized to AA $(n=50)$ or placebo $(n=50)$ using permuted blocks randomization. Enrollment was completed in a period of 6 months between 2016 and 2017. Follow-up visits took place at 6 months after treatment initiation.

The aim of the present study was to assess the effects of AA on glucose homeostasis in patients with prediabetes. The primary efficacy endpoint was the change in $\mathrm{HbA}_{1 \mathrm{c}}$ levels at 6 months after treatment initiation. Secondary endpoints included changes at 6 months in: i) fasting plasma glucose and insulin levels, ii) homeostasis model assessment-insulin resistance (HOMA-IR) index, iii) total cholesterol (TC), triglyceride, HDL-C and LDL-C levels, iv) basic biochemical parameters (serum creatinine, urea, uric acid, aminotransferases and creatine kinase (CK)), as well as the occurrence of any adverse effects associated with treatment.

\section{Laboratory measurements}

All laboratory measurements were carried out after an overnight fast (water consumption was allowed). Serum levels of fasting glucose, TC, HDL-C, triglycerides, uric acid, creatinine, urea, and CK, as well as serum activities of aspartate aminotransferase (AST), and alanine aminotransferase (ALT) were determined enzymatically in the laboratory of the University Hospital of loannina using an Olympus AU 600 analyzer (Olympus Diagnostica GmbH, Hamburg, Germany). LDL-C was calculated using the Friedewald equation. Fasting serum insulin was measured by an AXSYM insulin assay microparticle enzyme immunoassay on an AXSYM analyzer (Abbott Diagnostics, Illinois, USA). The HOMA-IR index was calculated as follows: HOMA-IR index = fasting insulin $(\mathrm{mU} / \mathrm{l}) \times$ fasting glucose $(\mathrm{mg} / \mathrm{dl}) / 405$. The determination of $\mathrm{HbA}_{1 \mathrm{c}}$ was based on a standardized latex agglutination inhibition assay (Randox Laboratories Ltd., Antrim, UK). HbA 1 values are expressed as percentage of total hemoglobin concentration. The sensitivity of the method is $0.25 \mathrm{~g} / \mathrm{dl}$ of $\mathrm{HbA}_{1 \mathrm{c}}$ and the within-run and between-run precision are $<6.67 \%$ and $<4.82 \%$, respectively. 


\section{Statistical analysis}

We used the statistical program G*Power 3.0.10 for power calculation. It was estimated that a sample size of 88 would give an $80 \%$ power to detect a $0.4 \%$ difference in the levels of $\mathrm{HbA}_{1 c}$ concentration at a 2 -sided $\alpha$ of 0.05 . We included 100 patients allowing for a drop-out rate of $\sim 10 \%$. All parameters were checked for normality with the Kolmogorov-Smirnov test and non-normally distributed variables were log-transformed. Data are expressed as the mean \pm SD except for non-normally distributed variables, which are presented as median (range). The paired-samples $t$-test and the Wilcoxon's rank test for normally distributed and non-normally distributed variables, respectively, were used to assess the effect of treatment in each group. Analysis of covariance (ANCOVA), adjusted for baseline values, was used for the comparisons between treatment groups. Significance was defined as $p<0.05$. All analyses were carried out with SPSS 16.0 (SPSS Inc.).

\section{Results}

We enrolled 100 patients (49 men and 51 women, mean age: $57 \pm 7$ years). Baseline characteristics of study participants are shown in Table I. No significant difference regarding baseline data, including drugs (data not shown), was found between groups. Overall, 87 patients completed the study; compliance rate was $>80 \%$ in these patients. Of the remaining 13 patients, 7 did not complete the 6-month follow-up, 1 withdrew informed consent and 5 discontinued treatment due to side effects (bad taste).

No significant change was observed at 6 months in either group in $\mathrm{HbA}_{1 c}$, fasting plasma glucose, insulin or HOMA-IR index. Furthermore, none of the lipid profile parameters was significantly altered in either treatment group. All changes are shown in Table II.

Measured biochemical parameters remained unaltered in both groups (not shown). No adverse effects were reported apart from bad taste after pill ingestion in patients $(n=5)$ who discontinued therapy.

\section{Discussion}

In this double-blind placebo-controlled study we demonstrated for the first time that treatment with a formulation containing extracts of bergamot, fenugreek and olive leaf for 6 months did not result in any change in glycemic or lipid profile in subjects with prediabetes.

A meta-analysis including 10 studies on the effect of fenugreek on glycemia demonstrated significant reductions in fasting glucose $(-0.96 \mathrm{mmol} / \mathrm{l}$; $17.3 \mathrm{mg} / \mathrm{dl}, 95 \%$ confidence interval $(\mathrm{Cl}):-1.52$, $-0.40 ; R^{2}=80 \% ; 10$ trials), $2 \mathrm{~h}$ post-load glucose (-2.19 mmol/l; $39.4 \mathrm{mg} / \mathrm{dl}, 95 \% \mathrm{Cl}:-3.19,-1.19$; $R^{2}=71 \% ; 7$ trials) and $\mathrm{HbA}_{1 c}(-0.85 \% ; 95 \% \mathrm{Cl}$ : $-1.49 \%,-0.22 \%$; $P^{2}=0 \%$; 3 trials) with fenugreek administration compared with control interventions [6]. On the other hand, the pooled effect of fenugreek on fasting serum insulin was not significant [6]. These results should be interpreted with caution for several reasons. Most of the included trials were of low methodological quality and none of them reported the methods of randomization or allocation concealment, while only a few trials provided information on blinding status and dropout rates. Furthermore, the sample size for all trials combined was 278 (range: 5-15 participants for

Table I. Anthropometric and metabolic parameters at baseline

\begin{tabular}{|c|c|c|c|}
\hline Parameter & $\mathrm{AA}(n=50)$ & Placebo $(n=50)$ & $P$-value \\
\hline Male/female & $23 / 27$ & $26 / 24$ & NS \\
\hline Smoking (\%) & 23 & 28 & NS \\
\hline Body weight $[\mathrm{kg}]$ & $83.2 \pm 20$ & $82.9 \pm 16$ & NS \\
\hline Body mass index (BMI) $\left[\mathrm{kg} / \mathrm{m}^{2}\right]$ & $29.0 \pm 3.0$ & $30.4 \pm 5.8$ & NS \\
\hline Systolic blood pressure [mm Hg] & $126 \pm 14$ & $125 \pm 12$ & NS \\
\hline Diastolic blood pressure [mm Hg] & $76 \pm 9$ & $76 \pm 9$ & NS \\
\hline Fasting plasma glucose $[\mathrm{mg} / \mathrm{dl}]$ & $105.4 \pm 8.7$ & $102.6 \pm 7.0$ & NS \\
\hline Fasting plasma insulin $[\mu \mathrm{U} / \mathrm{ml}]$ & $10.2(1.6-4.7)$ & $14(6.0-25.4)$ & NS \\
\hline HOMA-IR index & $2.4(0.1-12.0)$ & $2.5(0.3-11.0)$ & NS \\
\hline $\mathrm{HbA}_{1 \mathrm{c}}(\%)$ & $5.9 \pm 0.3$ & $5.9 \pm 0.3$ & NS \\
\hline Total cholesterol [mg/dl] & $164 \pm 21$ & $165 \pm 26$ & NS \\
\hline Triglycerides [mg/dl] & $121(57-271)$ & $121(64-256)$ & NS \\
\hline High-density lipoprotein cholesterol [mg/dl] & $52 \pm 9$ & $49 \pm 11$ & NS \\
\hline Low-density lipoprotein cholesterol [mg/dl] & $88 \pm 18$ & $93 \pm 21$ & NS \\
\hline
\end{tabular}


Table II. Change of laboratory values at 6 months

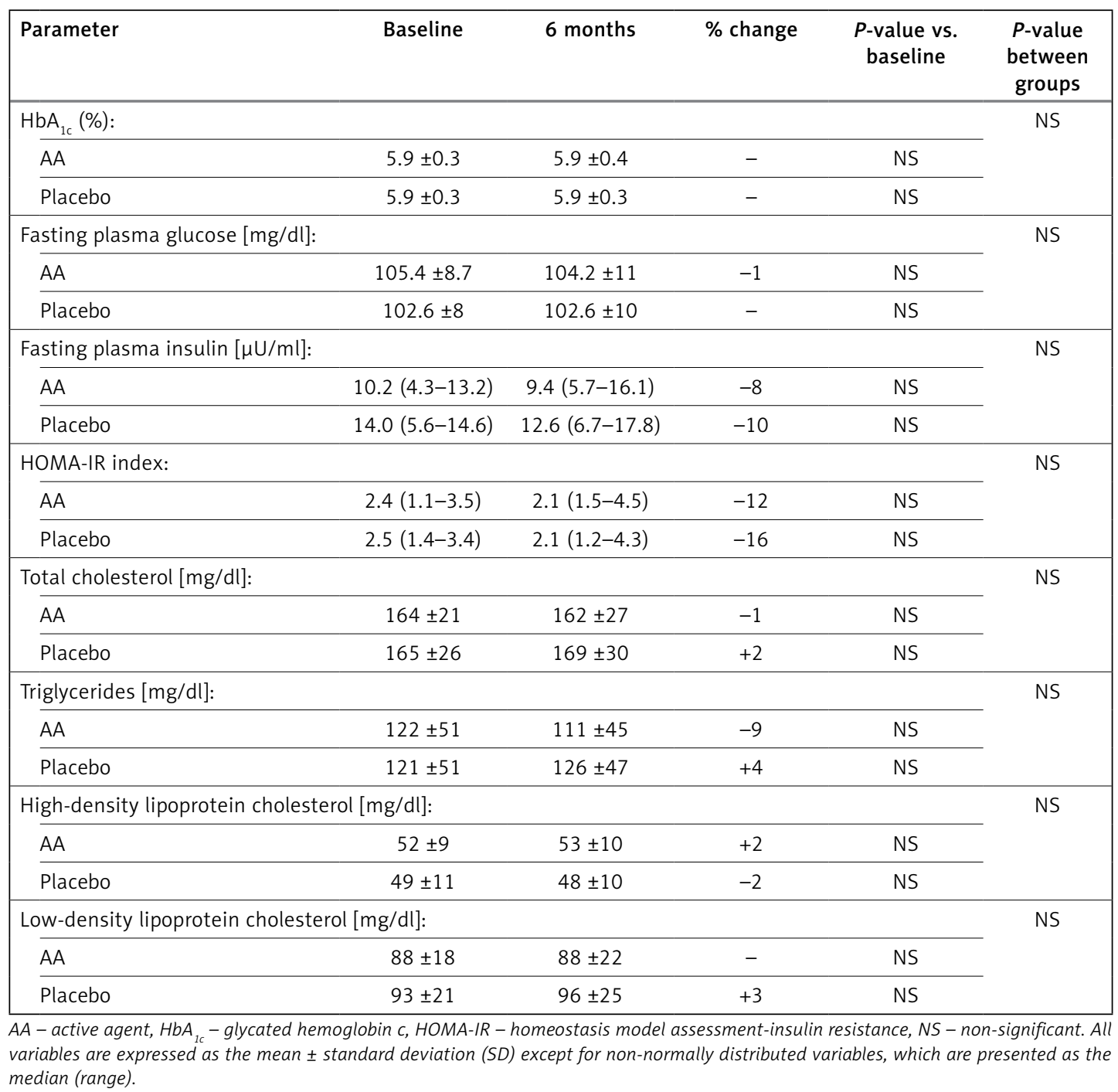

crossover trials and 25-69 participants for parallel trials; thus, the findings are derived only from small studies). There was a wide range in the daily dose of fenugreek seed, i.e. 1-100 g (median: $25 \mathrm{~g}$ ), and study duration (10-84 days; median: 30 days) [6]. Obviously, there is considerable heterogeneity in study results depending on diabetes status and fenugreek dose. In fact, significant effects on fasting and $2 \mathrm{~h}$ postprandial glucose were only observed in studies which included patients with T2D and used medium or high doses of fenugreek [6]. Most of the studies included in this meta-analysis involved T2D patients treated with diet or oral anti-diabetic agents. One study was conducted in subjects with T1D and only 2 studies included non-diabetic participants. We could assume that the neutral effects seen in our study may be due to the inclusion of only prediabetic individuals. Of note, baseline fasting glucose and $\mathrm{HbA}_{1 \mathrm{c}}$ in our study were closer to normal than to diabetic values. Furthermore, the dose of fenugreek was only $200 \mathrm{mg} /$ day, which is much smaller than the doses used in most other studies. Nevertheless, our study was adequately powered, was randomized double-blind, placebo-controlled and had sufficient duration.

After the publication of the above mentioned meta-analysis, a randomized, double-blind, placebo-controlled study evaluated the efficacy and safety of standardized fenugreek seed extract (500 mg) in 154 patients with T2D [7]. The active treatment was associated with significant reductions in $\mathrm{HbA}_{1 \mathrm{c}}(-18 \%)$, fasting $(-22 \%)$ and postprandial plasma (PP) glucose levels (-31\%), as well as an increase in C-peptide levels compared with baseline. Furthermore, in the fenugreek group a reduction in concomitant anti-diabetic medications was observed. We should note, though, that significant reductions of $17.4 \%$ and $16.0 \%$ in PP glucose and $\mathrm{HbA}_{1 \mathrm{c}}$, respectively, were observed in the placebo group as well; however, the difference between groups was significant. Therefore, the overall beneficial effects of fenugreek in this study 
may not be attributed only to the agent itself, but rather to other factors, e.g. adherence to lifestyle in the context of the close monitoring of a clinical trial. The design of this study was of higher quality compared with the ones included in the aforementioned meta-analysis. The discrepancy between the findings of this and our study may be attributed to the different patient populations, as previously discussed. It can be speculated that fenugreek improves glucose metabolism only if it is severely impaired (i.e. the greater the baseline $\mathrm{HbA}_{1 \mathrm{c}}$, the greater the reduction achieved). Furthermore, the dose of fenugreek used in this study was 2.5 times higher than the one we used.

We found only one study in non-diabetic participants that was not included in the aforementioned meta-analysis. Fourteen overweight and obese Asian subjects 32-52 years old received food with a high glycemic index and were randomized to treatment with fenugreek or no treatment. The primary endpoint was the incremental area under the plasma glucose response curve (IAUC) [5]. Adding fenugreek to foods significantly reduced the IAUC compared with food alone. This study demonstrated a favorable effect of fenugreek in glucose homeostasis, which is in contrast with the results of our study. However, the patient population had several differences compared with ours, i.e. it included Asian subjects and subjects of younger age. Furthermore, the sample size was relatively small and the study was not randomized, double-blind, placebo-controlled.

We should note that the other extracts (from bergamot and olive leaf) included in the AA have not been associated with deleterious effects on glycemic profile [8] and, thus, cannot be blamed for the neutral effect of AA in glucose metabolism parameters. To the contrary, olive leaf polyphenols may improve insulin sensitivity and pancreatic $\beta$-cell responsiveness [8], while there is evidence that bergamot extract has beneficial effects in hyperglycemia [9].

Regarding lipid profile, we did not observe any significant alterations in any of the lipid parameters. Importantly, almost all participants were on statin and/or other lipid lowering treatment and most of them were adequately controlled. Therefore, it would not be easy to show additional lipid lowering with this agent. In one study which showed improvement in all lipid parameters with bergamot, patients had moderate hypercholesterolemia, but were statin-intolerant and received no lipid-lowering agent [10].

In conclusion, an agent containing fenugreek, bergamot and olive leaf extracts in patients with prediabetes was not associated with any change in glycemic or lipid profile. Future studies may shed more light on the role of these substances in patients with overt T2D.

\section{Acknowledgments}

The pills with $A A$ and placebo were kindly supplied by FARAN SA., which had no role in the design of the study, data interpretation or manuscript writing.

\section{Conflict of interest}

The authors declare no conflict of interest.

\section{References}

1. Forouhi NG, Wareham NJ. Epidemiology of diabetes. Medicine 2014; 42: 698-702.

2. Yeboah J, Bertoni AG, Herrington DM, Post WS, Burke GL. Impaired fasting glucose and the risk of incident diabetes mellitus and cardiovascular events in an adult population: MESA (Multi-Ethnic Study of Atherosclerosis). J Am Coll Cardiol 2011; 58: 140-6.

3. Prevention or delay of type 2 diabetes: standards of medical care in diabetes-2019. Diabetes Care 2019; 42: S29-33.

4. Heianza Y, Hara S, Arase Y, et al. HbA1c $5.7-6.4 \%$ and impaired fasting plasma glucose for diagnosis of prediabetes and risk of progression to diabetes in Japan (TOPICS 3): a longitudinal cohort study. Lancet 2011; 378: 147-55.

5. Robert SD, Ismail AA, Wan Rosli WI. Trigonella foenum-graecum seeds lowers postprandial blood glucose in overweight and obese individuals. J Nutr Metab 2014; 2014: 964873.

6. Neelakantan N, Narayanan M, de Souza RJ, van Dam RM. Effect of fenugreek (Trigonella foenum-graecum L.) intake on glycemia: a meta-analysis of clinical trials. Nutr J 2014; $13: 7$

7. Verma N, Usman K, Awasthi V, Goel PK, Lamgora G. Clinical evaluation fenugreek seed extract in patients with type-2 diabetes: an add-on study in 154 patients. WJPR 2015; 4: 2266-79.

8. de Bock M, Derraik JG, Brennan CM, et al. Olive (Olea europaea L.) leaf polyphenols improve insulin sensitivity in middle-aged overweight men: a randomized, placebo-controlled, crossover trial. PLoS One 2013; 8: e57622.

9. Mollace V, Sacco I, Janda E, et al. Hypolipemic and hypoglycaemic activity of bergamot polyphenols: from animal models to human studies. Fitoterapia 2011; 82: 309-16.

10. Toth PP, Patti AM, Nikolic D, et al. Bergamot reduces plasma lipids, atherogenic small dense LDL, and subclinical atherosclerosis in subjects with moderate hypercholesterolemia: a 6 months prospective study. Front Pharmacol 2016; 6: 299. 